\title{
Immunoproteasome dysfunction augments alternative polarization of alveolar macrophages
}

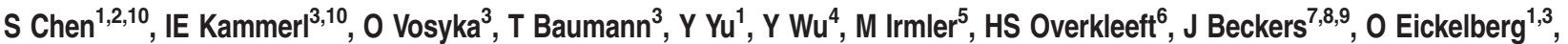 \\ $\mathrm{S}$ Meiners ${ }^{*, 3}$ and T Stoeger, ${ }^{*}$
}

The proteasome is a central regulatory hub for intracellular signaling by degrading numerous signaling mediators. Immunoproteasomes are specialized types of proteasomes involved in shaping adaptive immune responses, but their role in innate immune signaling is still elusive. Here, we analyzed immunoproteasome function for polarization of alveolar macrophages, highly specialized tissue macrophages of the alveolar lung surface. Classical activation (M1 polarization) of primary alveolar macrophages by LPS/IFN $\gamma$ transcriptionally induced all three immunoproteasome subunits, low molecular mass protein 2 (LMP2), LMP7 and multicatalytic endopeptidase complex-like 1, which was accompanied by increased immunoproteasome activity in M1 cells. Deficiency of LMP7 had no effect on the LPS/IFN $\gamma$-triggered M1 profile indicating that immunoproteasome function is dispensable for classical alveolar macrophage activation. In contrast, IL-4 triggered alternative (M2) activation of primary alveolar macrophages was accompanied by a transcriptionally independent amplified expression of LMP2 and LMP7 and an increase in immunoproteasome activity. Alveolar macrophages from LMP7 knockout mice disclosed a distorted M2 profile upon IL-4 stimulation as characterized by increased M2 marker gene expression and CCL17 cytokine release. Comparative transcriptome analysis revealed enrichment of IL-4-responsive genes and of genes involved in cellular response to defense, wounding and inflammation in LMP7-deficient alveolar macrophages indicating a distinct M2 inflammation resolving phenotype. Moreover, augmented M2 polarization was accompanied by amplified AKT/STAT6 activation and increased RNA and protein expression of the M2 master transcription factor interferon regulatory factor 4 in LMP7 ${ }^{-1-}$ alveolar macrophages. IL-13 stimulation of LMP7-deficient macrophages induced a similar M2-skewed profile indicative for augmented signaling via the IL-4 receptor $\alpha$ (IL4R $\alpha$ ). IL4R $\alpha$ expression was generally elevated only on protein but not RNA level in LMP7 ${ }^{-l-}$ alveolar macrophages. Importantly, specific catalytic inhibition with an LMP7-specific proteasome inhibitor confirmed augmented IL-4-mediated M2 polarization of alveolar macrophages. Our results thus suggest a novel role of immunoproteasome function for regulating alternative activation of macrophages by limiting IL4R $\alpha$ expression and signaling.

Cell Death and Differentiation (2016) 23, 1026-1037; doi:10.1038/cdd.2016.3; published online 18 March 2016

The immunoproteasome is a specialized type of proteasome and constitutively active in immune cells, whereas standard proteasomes are expressed in all non-immune cells. Similar to the standard $20 \mathrm{~S}$ proteasome, immunoproteasomes are composed of two outer $a$-rings and two inner $\beta$-rings of seven subunits each ${ }^{1}$ but contain a different set of IFN $\gamma$-inducible

${ }^{1}$ Comprehensive Pneumology Center (CPC), Institute of Lung Biology and Disease, Helmholtz Zentrum München, Neuherberg, Germany; ${ }^{2}$ Department of Pathophysiology, West China School of Preclinical Sciences and Forensic Medicine, Sichuan University, Chengdu, Sichuan Province, China; ${ }^{3}$ Comprehensive Pneumology Center (CPC), University Hospital, Ludwig-Maximilians University and Helmholtz Zentrum München, Member of the German Center for Lung Research (DZL), Munich, Germany; ${ }^{4}$ Max von Pettenkofer-Institute, Ludwig-Maximilians University, Munich, Germany; ${ }^{5}$ Institute of Experimental Genetics, Helmholtz Zentrum München, Neuherberg, Germany; ${ }^{6}$ Department of Bio-Organic Synthesis, Leiden University, Leiden, The Netherlands; ${ }^{7}$ Institute of Experimental Genetics, Helmholtz Zentrum München GmbH, Neuherberg, Germany; ${ }^{8}$ German Center for Diabetes Research (DZD e.V.), Neuherberg, Germany and ${ }^{9}$ Technische Universität München, Chair of Experimental Genetics, Freising, Germany

*Corresponding author: S Meiners, Comprehensive Pneumology Center (CPC), University Hospital, Ludwig-Maximilians University and Helmholtz Zentrum München, Member of the German Center for Lung Research (DZL), München 81377, Germany. Tel: +49 89 31874673; Fax: +49 89 3187-4661;

E-mail: silke.meiners@helmholtz-muenchen.de

or T Stöger, Comprehensive Pneumology Center (CPC), Institute of Lung Biology and Disease, Helmholtz Zentrum München, Ingolstädter Landstrasse 1, Neuherberg D-85764, Germany. Tel: +49 89 31873104; Fax: +49 89 3187-2400; E-mail: tobias.stoeger @helmholtz-muenchen.de

${ }^{10}$ These authors contributed equally to this work.

Abbreviations: ABPS, activity-based probes; Actb, actin, beta; AKT, thymoma viral proto-oncogene 1 (RAC-alpha serine/threonine-protein kinase); ARDS, Acute respiratory distress syndrome; Arg1, arginase, liver; BAL, Bronchoalveolar lavage; Ccl17 /18, chemokine (C-C motif) ligand 17/18; Ccr5, chemokine (C-C motif) receptor 5; Chchd10, coiled coil-helix-coiled-coil-helix domain containing 10; Cish, cytokine inducible SH2-containing protein; Clec10a, C-type lectin domain family 10, member A; DC, Dentritic cell; IFNg, interferon gamma; II12b, interleukin 12b; II1b, interleukin 1 beta; IIr1, interleukin 1 receptor, type I; IL4/10/13, interleukin 4/10/13; IL4R $\alpha$, IL4 receptor $\alpha$; Irf4/5, interferon regulatory factor 4/5; LMP2/7, low molecular mass protein 2/7; LPS, Lipopolysaccharide (Endotoxin); MECL-1, multicatalytic endopeptidase complex-like 1; Mrc1, mannose receptor, C type 1; Nfkb1, nuclear factor of kappa light polypeptide gene enhancer in B cells 1, p105; Nos2, nitric oxide synthase 2, inducible; Psma3/5/6/7/ 8/9/10, proteasome (prosome, macropain) subunit, alpha type 3/5/6/7/8/9/10; Ptgs1, prostaglandin-endoperoxide synthase 1; Pirb, paired Ig-like receptor B (PIR-B); Rela, v-rel reticuloendotheliosis viral oncogene homolog A (avian); Retnla, resistin like alpha; Rnase2a, ribonuclease, RNase A family, 2A (liver, eosinophil-derived neurotoxin); Sema4b, sema domain, immunoglobulin domain (Ig), transmembrane domain (TM) and short cytoplasmic domain, (semaphorin) 4B; STAT, signal transducer and activator of transcription; Tfrc, transferrin receptor; Th1/2, type 1/2 T helper cells; Tnf, tumor necrosis factor; Wt, wild type

Received 06.8.15; revised 22.12.15; accepted 11.1.16; Edited by X Lu; published online 18.3.16 
catalytic subunits, that is, low molecular mass protein (LMP) 2 , multicatalytic endopeptidase complex-like 1 (MECL-1) and LMP7 that substitute $\beta 1, \beta 2$ and $\beta 5$, respectively. ${ }^{2}$ Immunoproteasomes generate antigenic peptides for improved presentation on $\mathrm{MHC}$ I molecules compared with the standard proteasome, ${ }^{2,3}$ thereby mounting efficient immune surveillance by $\mathrm{CD}^{+} \mathrm{T}$ cells. Specific inhibition of the catalytic activity of LMP7 with a novel proteasome inhibitor revealed an extended function for immunoproteasomes in autoimmunity. ${ }^{2,4-6}$ Studies with immunoproteasome subunit knockout mice indicated that immunoproteasomes also regulate composition and activation of lymphocyte subsets and maturation and differentiation of B and Th17 cells, which may relate to the altered transcriptional profile and cytokine expression of immunoproteasome-deficient immune cells. ${ }^{7-10}$ These data suggest that immunoproteasome function affects both adaptive and also innate immune cell homeostasis. ${ }^{11,12}$

We here investigated immunoproteasome function in alveolar macrophages, which in the lungs express particularly high levels of immunoproteasomes. ${ }^{13}$ These cells are highly specialized tissue-resident macrophages that colonize the respiratory surface of the lung and are essential for the pulmonary host defense against inhaled pathogens but also for resolution of tissue damage. ${ }^{14-17}$

Similar to the well-studied bone marrow-derived macrophages, alveolar macrophages are highly plastic within a multi-dimensional spectrum of pro- and anti-inflammatory activation states depending on the cytokine milieu. ${ }^{18}$ Upon acute infection or injury, alveolar macrophages are activated into a classically activated M1 phenotype, and potently secrete pro-inflammatory cytokines to initiate recruitment of inflammatory cells. ${ }^{19} \mathrm{M} 1$ polarization involves LPS/IFN $\gamma$-mediated activation of pro-inflammatory transcription factors promoting expression of classical M1 marker genes and enhanced pathogen phagocytosis. Following the acute inflammatory burst, however, alveolar macrophages can remove cell debris and apoptotic inflammatory cells by so-called efferocytosis and polarize into alternatively activated M2 macrophages. ${ }^{20}$ In response to IL-4/-13 or IL-10 signaling, alveolar macrophages then acquire an anti-inflammatory state characterized by metabolic changes, activation of anti-inflammatory signaling factors, expression of M2 marker genes and the production of anti-inflammatory and growth-promoting cytokines. ${ }^{21-23}$ Otherwise, at certain disease conditions, M2-derived repair factors may also promote Th2 inflammatory settings and may thus have profibrotic activity. ${ }^{24-26}$

Here, we show that alternative M2 but not classical M1 activation is regulated by the immunoproteasome as revealed by augmented M2 polarization of alveolar macrophages upon genetic deficiency of LMP7 and specific catalytic inhibition of LMP7.

\section{Results}

Induction of active immunoproteasomes upon alveolar macrophage activation. In a first step, we characterized immunoproteasome expression during $\mathrm{M} 1$ and $\mathrm{M} 2$ polarization of murine alveolar macrophages from different mouse strains (BALB/C or C57BL/6) and the SV40-immortalized
BALB/c-derived alveolar macrophage cell line MH-S. Cells were treated for $24 \mathrm{~h}$ either with LPS and IFN $\gamma$ to induce M1-like phenotypes or with IL-4 for induction of M2 polarization. For all three sources of alveolar macrophages, we observed typical changes in the alveolar macrophage morphology with globular M1-polarized cells and flat adherent M2-polarized cells (Supplementary Figure S1A), as well as the well-known differential RNA (Supplementary Figures S1B and $\mathrm{C}$ ) and protein (Supplementary Figure S1D) expression pattern for the respective polarization states. ${ }^{21,22,27}$

LPS/IFN $\gamma$-triggered M1 polarization uniformly upregulated RNA expression of the immunoproteasome subunits LMP2, MECL-1 and LMP7 in all three alveolar macrophage sources (Figure 1a). Kinetic analysis revealed markedly elevated RNA expression up to $72 \mathrm{~h}$ with slight differences in the extent of regulation for LPS and IFN $\gamma$ (Supplementary Figures S2A and B) In contrast, no significant changes were observed for IL-4-mediated M2 polarization even after $72 \mathrm{~h}$ (Figure 1a, Supplementary Figures S2A and B).

Western blot analysis confirmed pronounced upregulation of LMP2 and LMP7 in M1-polarized cells compared with unstimulated controls after $24 \mathrm{~h}$ (Figure 1b). Expression increased further up to $72 \mathrm{~h}$ (Supplementary Figure S2C). To our surprise, we also observed significantly elevated levels of LMP2 and in particular of LMP7 after $24 \mathrm{~h}$ of M2 polarization of primary alveolar macrophages (Figure 1b). Upregulation was even more pronounced after $72 \mathrm{~h}$ of IL-4 treatment (Supplementary Figure S2C).

Of interest, protein and also RNA expression of the $20 \mathrm{~S}$ $a$-subunits were induced to some extent in both M1- and M2-polarized cells (Figure 1b, Supplementary Figure S2D). To further corroborate the existence of catalytically active immunoproteasomes in polarized alveolar macrophages, we used a set of fluorescently labeled activity-based probes (ABPs) with distinct binding specificities to the different active sites. These ABPs covalently bind to the active-site threonine of the respective catalytic subunits and active subunits are quantified after separation of native cell extracts by SDS-PAGE (Figure 1c): Both, immunoproteasome and standard proteasome activities were significantly elevated in M1-polarized alveolar macrophages compared with the control. In M2-polarized macrophages, immunoproteasome activities were only slightly higher consistent with the relatively small increase in expression level after $24 \mathrm{~h}$ compared with $72 \mathrm{~h}$ of IL-4 treatment (Supplementary Figure S2C). We also observed elevated activities of the standard 205 subunits $\beta 1$ and $\beta 2$ and of $\beta 5 /$ LMP7 subunits in both M1- and M2-polarized macrophages, which also accords to our protein expression data.

LMP7 deficiency augments M2 macrophage polarization. To investigate, whether immunoproteasome activity of alveolar macrophages is of any functional relevance for macrophage polarization, we polarized primary cells from wild-type (wt) and $\mathrm{LMP}^{-/-}$mice toward $\mathrm{M} 1$ or $\mathrm{M} 2$ phenotypes, respectively. LMP7 disruption did not affect viability during polarization (Supplementary Figure S3A) and had no effect on M1 marker gene expression such as Nos2, Tnf, //1b and I/12b (Figure 2a). In accordance, gene expression levels of some of the transcriptional activators of 
a

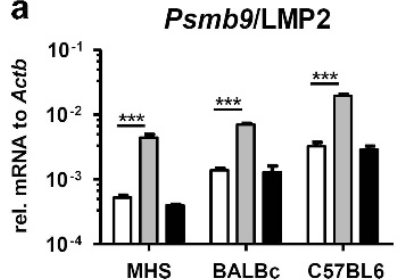

b
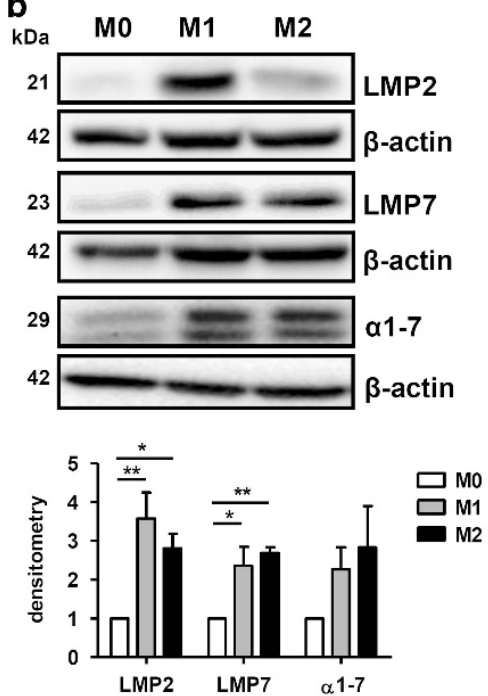

Psmb10/MECL-1

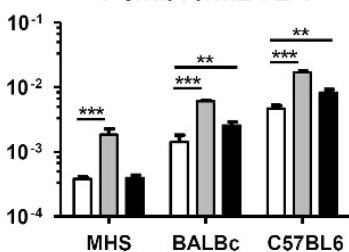

C
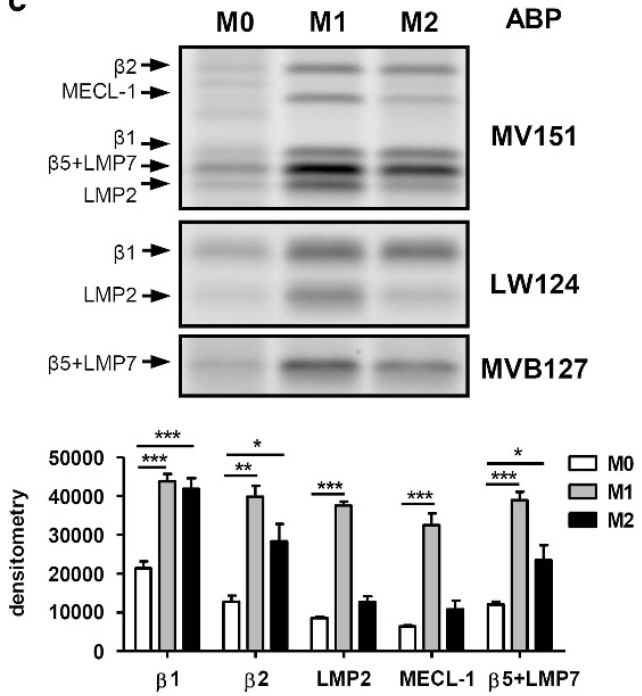

Figure 1 Expression of immunoproteasome subunits LMP2 and LMP7 is induced in M1- and M2-polarized alveolar macrophages. (a) Immunoproteasome subunit gene expression in MH-S cells or primary alveolar macrophages from BALB/C or C57BL/6 polarized into M1 or M2 for $24 \mathrm{~h}$ by stimulation with LPS/IFN $\gamma$ or IL-4, respectively. Nonpolarized cells (M0) served as controls: Psmb9 (LMP2), Psmb10 (MECL-1) and Psmb8 (LMP7) RNA expression is displayed relative to Actb. Results are combined data from at least three independent experiments (mean + S.E.M.). (b) Immunoproteasome subunit LMP2 and LMP7 protein expression as well as 20S $\alpha 1-7$ subunits in primary alveolar macrophages from C57BL/6 mice polarized for $24 \mathrm{~h}$. Results are representative for three independent experiments, densitometric analysis shows the fold-increase over M0 in three different experiments (mean +S.E.M.). (c) Proteasome activity in polarized alveolar macrophages from C57BL/6 mice detected after $48 \mathrm{~h}$ stimulation by ABPs MV151 (labeling all catalytically active $\beta$-subunits), LW124 ( $\beta 1$ and LMP2) or MVB127 ( $\beta 5$ and LMP7). Densitometric analysis displays the combined data from three individual experiments (mean + S.E.M.) loaded on the same gel. Statistical analysis (a)-(c): One-way ANOVA with Dunnetts's multiple comparison test (all columns versus control column (M0), $\left.{ }^{*} P<0.05,{ }^{* \star} P<0.01,{ }^{* \star *} P<0.001\right)$

M1 polarization such as Nfkb1, Rela and Irf5 were not altered by LMP7 deficiency (Supplementary Figure S3B). Intriguingly, LMP7 deficiency enhanced the IL-4 effect on alternative M2 polarization of alveolar macrophages (Figures $2 b$ and $c$ ). Gene expression of the major M2 marker Arg1, as well as the Th2 cytokine Ccl17 were clearly increased in $\mathrm{LMP}^{-1-}$ compared with wt cells. In addition, Retnla mRNA expression was considerably elevated in LMP7 ${ }^{-1-}$ cells, whereas Mrc1 expression was not altered. For Arg1 expression, this effect was already evident at lower doses of $1-5 \mathrm{ng} / \mathrm{ml} \mathrm{IL-4}$ (Supplementary Figure S3C). Secretion of CCL17 into supernatants of polarized alveolar macrophages was also significantly enhanced in M2-polarized cells from $\mathrm{LMP}^{-1-}$ mice as determined by ELISA. Taken together, our results clearly indicate that expression and activity of the immunoproteasome subunit LMP7 is of functional importance for alternative M2 activation of alveolar macrophages.

To comprehensively examine the impact of LMP7 deficiency on IL-4-induced M2 polarization, we performed comparative whole-transcriptome analysis for M0 and M2 alveolar macrophages from wt and LMP7 ${ }^{-1-}$ mice and focused mainly on genes upregulated by IL-4. Although wt and LMP7-deficient macrophages did not significantly differ in their transcriptome at the $\mathrm{MO}$ state, we observed a generally increased expression of IL-4-induced genes in M2-polarized alveolar macrophages from $\mathrm{LMP7}^{-1-}$ mice (Figure $3 \mathrm{a}$ and Supplementary Table SII). Among the most regulated genes were the already investigated major M2 markers Arg1, and Retnla as well as the M2 cytokine Ccl17. Quantitative RT-PCR analysis of an additional set of nine strongly upregulated genes (Rnase2a, Cish, Clec10a, Tfrc, Ptgs1, Chchd10, Sema4b, Ccr5 and I/1r1) confirmed our array results (Figure $3 b$ ). Moreover, gene ontology analysis for all genes that were upregulated in $\mathrm{LMP}^{-1-}$ M2 alveolar macrophages revealed significant enrichment of pathways related to the biological process 'defense response', 'response to wounding' and 'regulation of inflammatory response', indicative for a prominent M2 inflammation resolving phenotype of LMP7-deficient alveolar macrophages (Figure 3c).

Altered IL-4 signaling in LMP7-deficient macrophages. Gene set enrichment analysis (GSEA) for genes regulated by the IL-4 pathway revealed aggravated expression of several IL-4-induced genes in LMP7-deficient M2 alveolar 

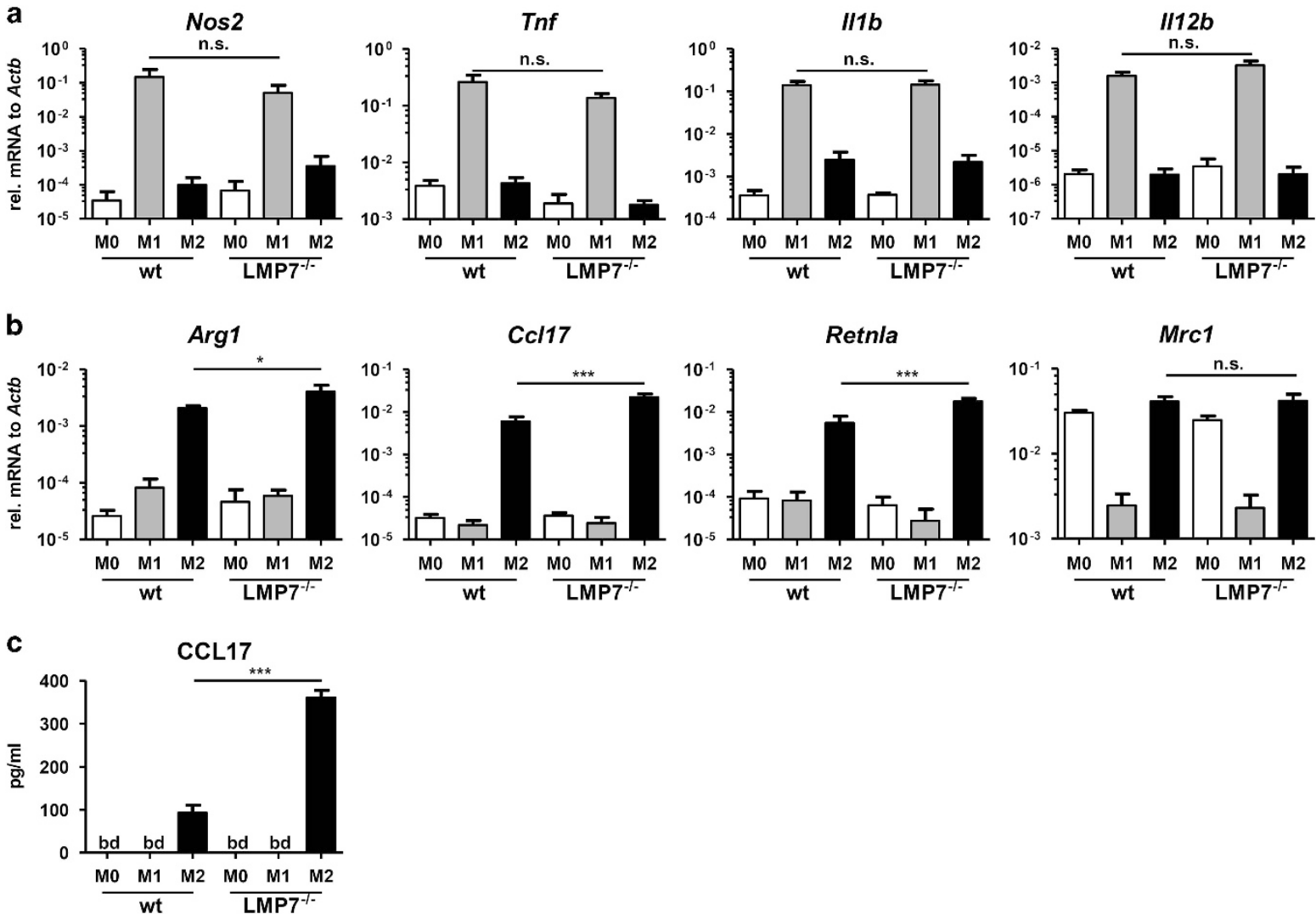

Figure 2 Deficiency of LMP7 immunoproteasome increases M2 alveolar macrophage polarization markers. (a) M1 marker gene expression analysis of primary alveolar macrophages from C57BL/6 wt or LMP7 ${ }^{-1}-$ mice polarized toward M1 phenotype (LPS and IFN $\gamma$ for $24 \mathrm{~h}$ ): expression of Nos2 (iNOS), Tnf, II1b, II12b is shown relative to Actb. Results are the combined data (mean+S.E.M.) from three independent experiments. (b) M2 marker gene expression analysis of primary alveolar macrophages from C57BL/6 wt or LMP7 ${ }^{-1-}$ mice polarized toward M2 phenotype (IL-4 for $24 \mathrm{~h}$ ): expression of Arg1 (Arginase 1), Ccl17, Retnla (Fizz-1), Mrc1 (mannose receptor 1) relative to Actb expression. Results are the combined data (mean+S.E.M.) from three independent experiments. (c) CCL17 secretion measured in supernatants of polarized primary alveolar macrophages from C57BL/6 wt or LMP7 ${ }^{-1-}$ mice (M1: LPS and IFN $\gamma$; M2: IL-4 for $24 \mathrm{~h}$ ). Results show the mean+S.E.M. from four individual mice and are representative for two independent experiments. Statistical analysis for (a) and (b): one-way ANOVA with Bonferroni's multiple comparison test; statistics for (c): Student's $t$-test; $\left({ }^{\star} P<0.05\right.$, $\left.{ }^{* \star \star} P<0.001\right)$. bd, below detection; n.s., not significant

macrophages supporting the concept of altered IL-4-mediated signaling in LMP7-deficient macrophages (Figure 4a, Supplementary Figure 4A). In the volcano plot, we identified the previously described key regulator of $\mathrm{M} 2$ macrophage polarization interferon regulatory factor 4 $(\text { IRF4 })^{28,29}$ as the only transcription factor among the most significantly altered genes (Figure $4 \mathrm{~b}$ ) being 2.5-fold induced by IL-4 in wt but 3.9 -fold in LMP7 ${ }^{-1-}$ cells, and finally also expressed at considerable levels (Supplementary Figure S4B). Increased expression of IRF4 in LMP7-deficient M2 macrophages was confirmed by qRT-PCR in a kinetic analysis from 6 to $72 \mathrm{~h}$ of IL-4 stimulation (Figure 4c). We also confirmed M2-specific mRNA induction of IRF4 in different primary cells from C57BL/6 or BALB/c mice and in the $\mathrm{MH}-\mathrm{S}$ cell line (Supplementary Figure $\mathrm{S4C}$ ). At the protein level, IRF4 increased up to $72 \mathrm{~h}$ only after IL-4 stimulation (Supplementary Figure S4D). Moreover, shortterm stimulation of alveolar macrophages with IL-4 revealed elevated levels of IRF4 after 180 min in LMP7-deficient cells (Figure 4d). This coincided with aggravated phosphorylation and thus activation of STAT6 and AKT, the two major signaling hubs for IL-4-induced M2 polarization (Figures 4e and $\mathrm{f}$, Supplementary Figures S4D and E). ${ }^{22,23}$

Our transcriptomic and signaling data thus corroborate that LMP7-deficient alveolar macrophages have intrinsic differences that govern a differential signaling response to IL-4 toward M2 macrophage polarization.

Altered IL-4 receptor expression in LMP7-deficient macrophages. As IL-4 induces M2 polarization via IL-4 receptor a (IL4Ra), which is also activated by $\mathrm{IL}-13,{ }^{30}$ we confirmed the involvement of IL-4 receptor activation using IL-13. IL-13 also induced LMP2 and LMP7 protein expression (Figure 5a) and triggered STAT6 phosphorylation and IRF4 expression in primary alveolar macrophages (Figure 5b). Again, $\mathrm{LMP}^{-1-}$ cells showed enhanced M2 polarization after IL-13 stimulation as evidenced by elevated Arg1 and Retnla expression (Figure $5 \mathrm{c}$ ). No significant alterations, however, could be detected for Mrc1 and Cc/17. To shed light on the enhanced IL-4R signaling activity in LMP7-deficient cells, we next investigated IL4Ra expression. Of note, although I/4ra mRNA expression was not affected by the 
a

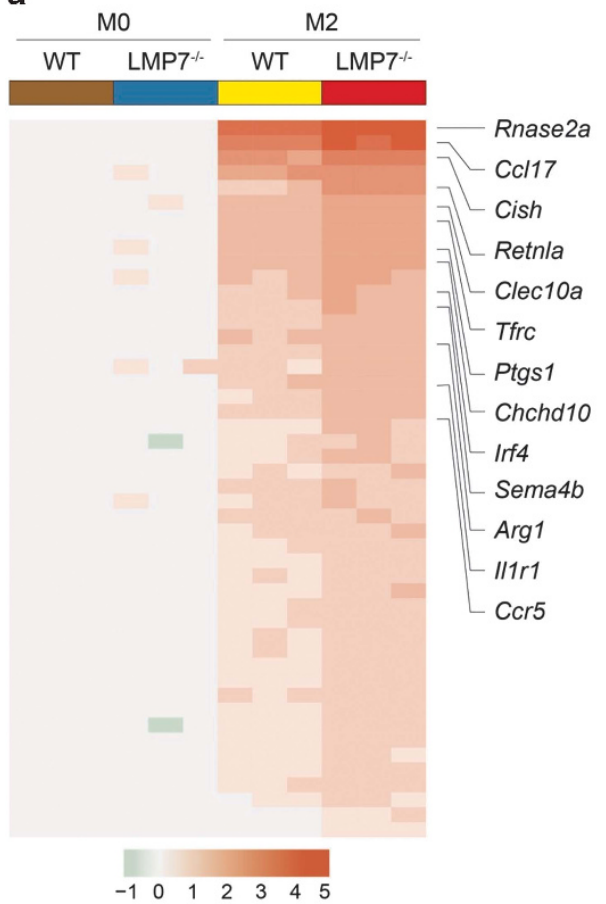

C

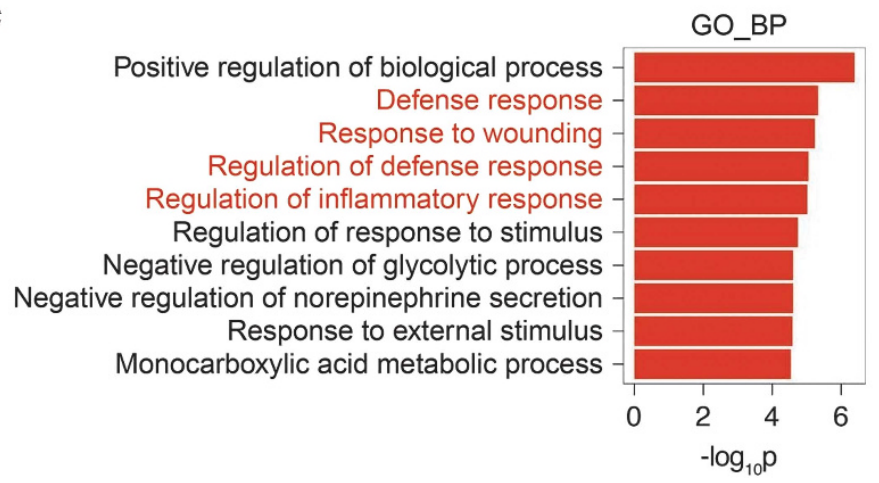

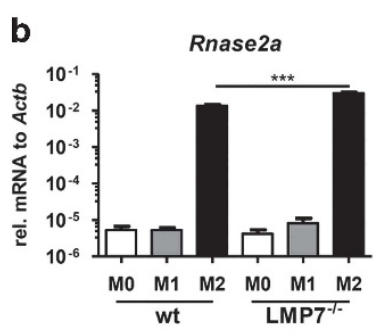
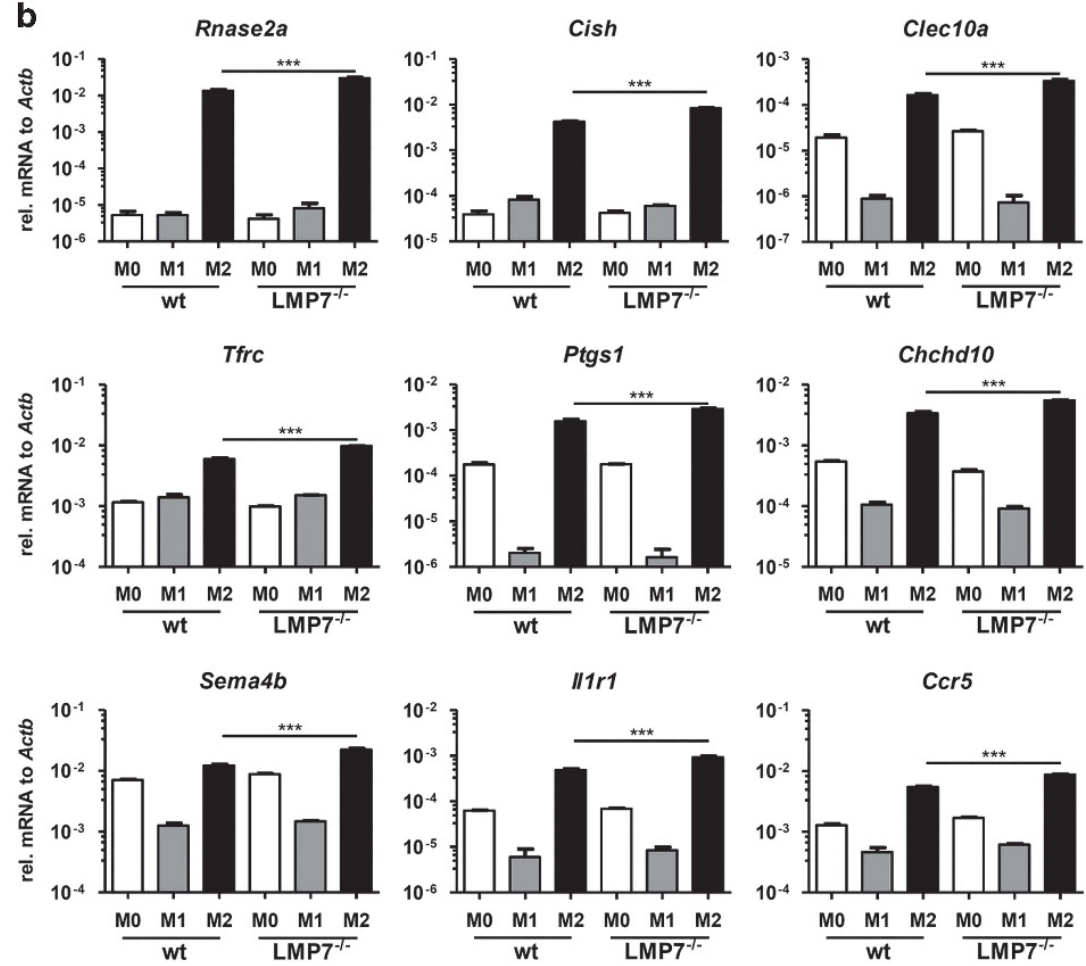

Figure 3 LMP7 deficiency affects M2 polarization at the transcriptome level. (a) Heatmap depicting genes that are significantly upregulated in LMP7 ${ }^{-1-}$ M2 compared with wt M2 (green, least expression; red, highest expression). (b) qPCR analysis of selected new M2 marker genes from the heat map: Rnase2a, Cish, Clec10a, Tfrc, Ptgs1, Chchd10, Sema4b, II1r1 and Ccr5 are shown relative to Actb. Results are the mean+S.E.M. from three individual C57BL/6 wt or LMP7 ${ }^{-1-}$ mice. (c) GO analysis: top GO terms enriched among genes that are upregulated in LMP7 ${ }^{-1-} \mathrm{M} 2$, relative to wt M2. One-way ANOVA with Bonferroni's multiple comparison test $\left({ }^{\star * *} P<0.001\right)$

genotype at any polarization condition (Figure 6a), IL4Ra protein expression was significantly elevated in $\mathrm{LMP}^{-1-}$ macrophages at baseline and in response to IL-4 stimulation compared with wt macrophages (Figure 6b). Elevated IL4Ra expression by LMP7-deficient alveolar macrophages thus represents a plausible explanation for augmented IL4R signaling and enhanced IL-4/-13-induced M2 polarization.

Inhibition of LMP7 activity augments alternative macrophage polarization. To confirm the role of the immunoproteasome subunit LMP7 for M2 polarization of alveolar macrophages and also to validate LMP7 as a drug target for modulation of IL-4 receptor signaling, we made use of the recently developed LMP7-specific immunoproteasome inhibitor ONX-0914. ${ }^{6,31}$ This inhibitor has been shown to discriminate between the standard $20 \mathrm{~S} \beta 5$ subunit and its immunoproteasome counterpart LMP7 and thus specifically inhibits LMP7. We first proved specificity of ONX-0914 for LMP7 using $\mathrm{MH}-\mathrm{S}$ cells and an ABP pulldown assay. ${ }^{32}$ Quantification of affinity-purified $\beta 5$ and LMP7 proteasome subunits by western blotting revealed that LMP7 was specifically lost by $50 \%$ and $80 \%$ when cells had been treated with 0.1 or $0.2 \mu \mathrm{M}$ ONX-0914, respectively, whereas $\beta 5$ was decreased only to a minor extent $(\sim 30 \%)$ at the highest dose of $1 \mu \mathrm{M}$ (Figure 7a). Specificity of ONX-0914 for the LMP7 immunoproteasome subunit was also confirmed by direct western blotting of cell lysates of ONX-0914-treated cells (Figure 7b). Here, inhibitor-bound versus unbound active subunits were differentiated by a mobility shift of LMP7 and $\beta 5$. 
a

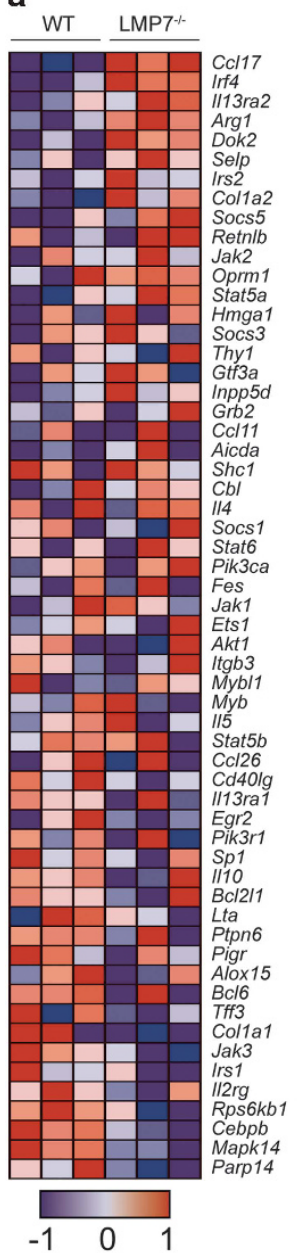

b

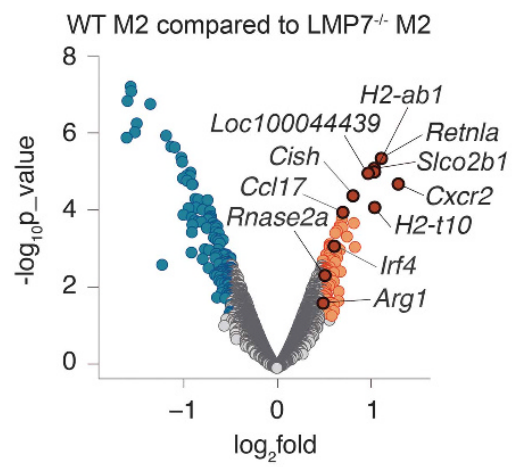

d
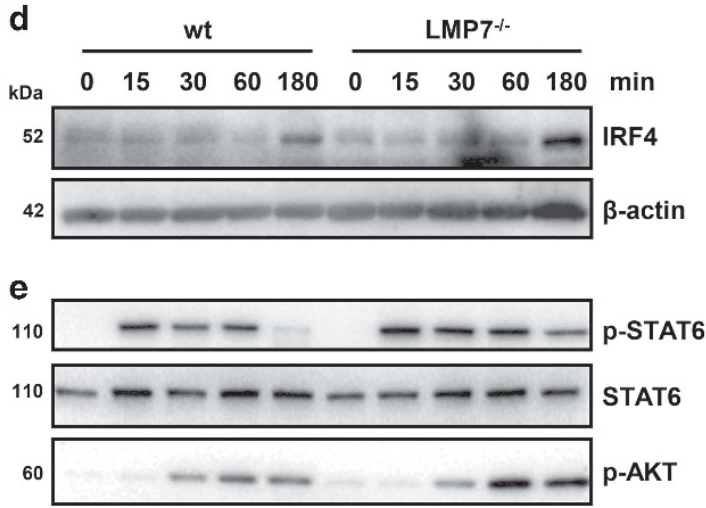

$600-m-m-m-m-m-m$ AKT

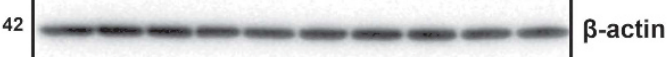

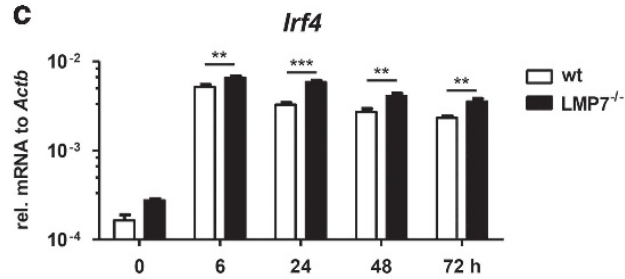

f
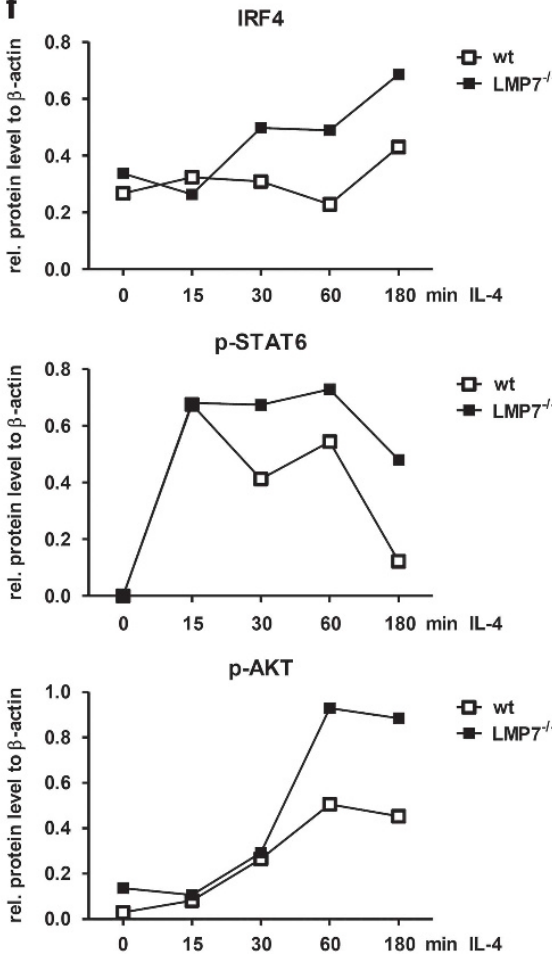

Figure 4 LMP7 deficiency augments alveolar macrophage M2 signaling. (a) Enrichment of IL-4 signaling according to Broad Institute (http://www.broadinstitute.org/) 'LU_IL4 signaling' related genes in LMP7 ${ }^{-1}$ M2 compared with wt M2 as shown by GSEA, IL-4 signaling. (b) Volcano plot showing genome-wide changes of gene expression in $\mathrm{LMP}^{-l-}$ M2 relative to wt M2. X axis represents the average log2 fold change, whereas y axis indicates the -log10P. Significantly changed genes $(P<0.05)$ with $x>0.5$ are represented by red circles and $x<0.5$ by blue circles. (c) Irf4 gene expression in primary alveolar macrophages from C57BL/6 wt or LMP7 ${ }^{-1-}$ mice treated with IL-4 for up to $72 \mathrm{~h}$. Results are combined data from three experiments (mean + S.E.M., two-way ANOVA with Bonferroni's post test, ${ }^{* \star} P<0.01$, ${ }^{* \star *} P<0.001$ ). (d) Time course of IRF4 (e) STAT6 $\left(\mathrm{pTyr}^{641}\right.$ ) and AKT (pSer ${ }^{473}$ ) pathway activation and expression within 0-180 min after IL-4 treatment in primary alveolar macrophages from C57BL/6 wt or $\mathrm{LMP7}^{-1-}$ mice. Results are representative for two independent experiments. (f) Densitometric analysis of protein levels of IRF4, p-STAT6 and p-AKT relative to $\beta$-actin for blots shown in (d) and (e)

In a next step, we treated MH-S cells with ONX-0914 and analyzed IL-4-mediated signaling and M2 marker gene expression. $\mathrm{MH}-\mathrm{S}$ cells were pretreated with 0.2 or $1 \mu \mathrm{M}$ ONX-0914, for a total of $5 \mathrm{~h}$ and stimulated with IL-4 for 180 to 15 min (see treatment scheme, Figure 8a). Very similar to the genetically depleted LMP7 macrophages, specific inhibition of LMP7 or enhanced IL-4-dependent STAT6 phosphorylation and resulted in elevated levels of IL4Ra (Figure 8b), which was most prominent at high inhibitor doses. Importantly, the LMP7-specific inhibitor significantly increased expression of the M2 polarization markers Arg1, Irf4 and Mrc1, which was particularly evident for $1 \mu \mathrm{M}$ of ONX-0914 and was reproducibly observed in primary alveolar macrophages (Figure 8c). In summary, our results reveal that LMP7 immunoproteasome activity is crucially involved in IL4R-driven alternative polarization of alveolar macrophages, most likely via limiting the expression of IL4Ra.

\section{Discussion}

In this study, we unravel a novel role for LMP7 immunoproteasome function for alternative activation of alveolar macrophages. We show for the first time that immunoproteasomes are induced by IL-4 receptor signaling in the course of M2 polarization via posttranscriptional mechanisms. Genetic ablation or specific catalytic inhibition of LMP7 augmented alternative activation of alveolar macrophages involving elevated IL4Ra expression, thereby enhancing their defense response profile. Our data thus suggest a novel role of immunoproteasome activity for regulating innate immune function of macrophages by limiting IL4R expression and signaling.

Expression and activity of immunoproteasomes in polarized alveolar macrophages. Intriguingly, formation of active immunoproteasomes was not only induced by 
a

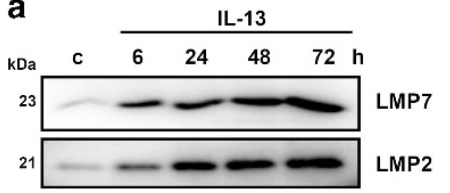

b

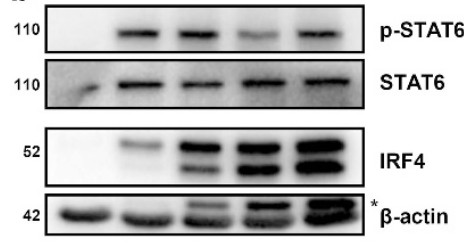

c
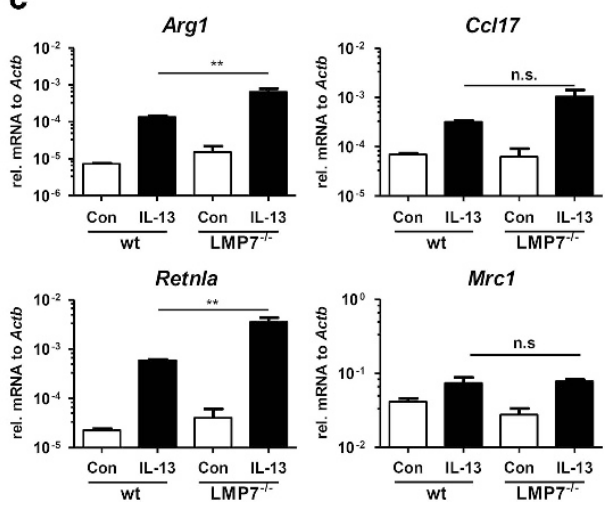

Figure $5 \mathrm{LL}-13$ treatment increases M2 polarization in LMP7 ${ }^{-1-}$ alveolar macrophages. (a) LMP2 and LMP7 protein expression in primary alveolar macrophages from C57BL/6 mice polarized for $24 \mathrm{~h}$ with IL-13. (b) STAT6 phosphorylation ( $\mathrm{pTyr}{ }^{641}$ ), total STAT6 expression and IRF4 expression up to $72 \mathrm{~h}$ after IL-13 treatment, $\beta$-actin expression served as loading control ( ${ }^{*}$ depicts residual IRF4 signal from first exposure). Results are representative for two independent experiments. (c) M2 marker gene expression Arg1, Ccl17, Retnla and Mrc1 in primary alveolar macrophages from three individual C57BL/6 wt or LMP7 ${ }^{-1-}$ mice (mean + S.E.M.). One-way ANOVA with Bonferroni's multiple comparison test; $\left({ }^{* *} P<0.01\right.$, n.s., not significant)

Th1-related stimuli like IFN $\gamma /$ LPS but also by Th2 cytokines, such as IL-4 and IL-13. Although transcriptional induction of immunoproteasomes by LPS and IFN $\gamma$ has been extensively described before for numerous immune and non-immune cell types $^{2,10-12}$ posttranscriptional upregulation and increased formation of active immunoproteasomes in response to IL-4 receptor signaling is a novel finding. IL-4 treatment of immature or mature dendritic cells (DC) did not result in increased expression and formation of immunoproteasomes but improved antigen presentation of $\mathrm{DCs}^{33}$ suggesting that activation of immunoproteasomes is a distinct feature of alternatively activated macrophages.

The delayed kinetics of standard and immunoproteasome activation with a steady increase in protein levels in $\mathrm{M} 1-$ or M2-polarized alveolar macrophages for up to $72 \mathrm{~h}$ suggest that upregulation of immunoproteasome activity is rather a consequence of the altered activation state of macrophages, as also suggested for DC differentiation. ${ }^{33}$ As we observed an overall activation of both standard and immunoproteasomes, it is tempting to speculate that increased proteasome activity is a general feature of cellular differentiation that helps to translate transcriptional reprogramming into differential protein content as suggested previously by us. ${ }^{34}$

Immunoproteasome function regulates IL-4 receptor signaling and alternative macrophage activation. The absence of the immunoproteasome subunit LMP7 augmented the ability of alveolar macrophages to respond to IL-4 and to polarize toward the M2 phenotype, but did not affect classical M1 activation. Accordingly, the M1 expression profile of well-known pro-inflammatory transcription factors of classical macrophage activation, for example, NF- $\kappa \mathrm{B} 1$ (p50/p65) and Irf5, was not altered in LMP7-depleted cells. Although the role of immunoproteasomes for canonical NF- $\kappa$ B1 signaling has been controversially discussed, ${ }^{35}$ we conclude that LMP7 is not required for p50/p65 signaling during M1 polarization of alveolar macrophages.

Remarkably, M2 polarization of alveolar macrophages was significantly disturbed by LMP7 immunoproteasome deficiency. Alternative macrophage activation was clearly enhanced in $\mathrm{LMP}^{-/-}$macrophages, as indicated by an amplified expression of the well-established M2 markers Arg1 and Retnla, and release of the IL-4-dependent Th2 chemokine CCL17. Furthermore, our microarray analysis revealed that LMP7 deficiency causes extensive alterations in the transcriptome of M2-polarized cells. Intriguingly, the majority of IL-4-inducible genes, which were found to be overrepresented in LMP7-deficient cells as compared with wt M2 alveolar macrophages, are well known for their involvement in pathways of defense, response to wounding and regulation of inflammation.

Our observation that LMP7 activity regulates M2 polarization was further corroborated by analysis of hallmark signaling mediators of IL-4. In particular, IL-4-induced expression of the M2-specific transcription factor IRF4 - a key transcription factor that controls M2 macrophage polarization ${ }^{28}$ - was already elevated at baseline levels and exceeded wt levels for up to 3 days upon LMP7 depletion. Similarly, STAT6 and AKT activation were both prolonged and enhanced in LMP7-deficient cells. As a very similar augmented M2 activation was observed for IL-13 stimulation of $\mathrm{LMP}^{-/-}$ cells, our data point toward altered IL-4 receptor signaling in LMP7-deficient macrophages. Although RNA expression of IL4R $a$ was not altered, protein expression was found to be significantly enhanced in LMP7 ${ }^{-/-}$cells already at baseline and thus most likely contributes to the amplified IL-4 and IL-13 signaling in $\mathrm{LMP}^{-/}$alveolar macrophages. IL4Ra turnover and ubiquitination-mediated proteasomal degradation have previously been described as an important mechanism limiting alternative $\mathrm{M} 2$ activation and airway inflammation, ${ }^{36}$ however, a specific involvement of the immunoproteasome for degradation of IL4Ra has not been recorded yet.

As deficiency of LMP7 is compensated by incorporation of the respective $\beta 5$ standard proteasome subunit, LMP7-deficient animals most likely assemble mixed or intermediate types of proteasome. These have been shown to have altered enzyme kinetics that may account for the distinct phenotypes of the single immunoproteasome 

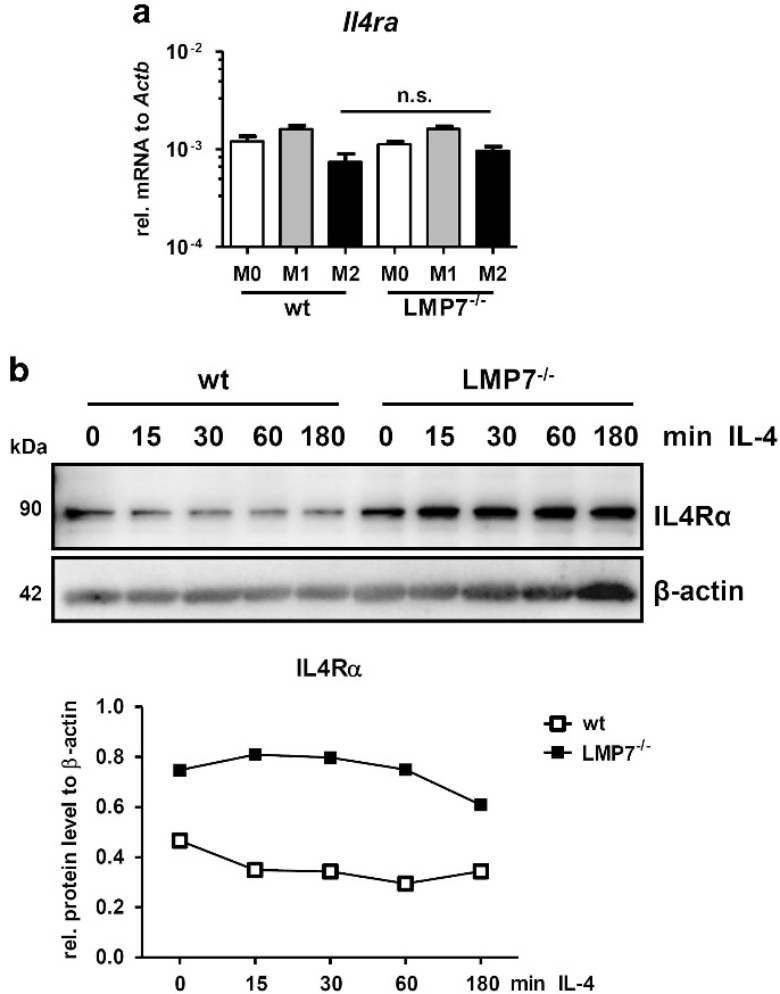

Figure 6 Effect of LMP7 deficiency on IL4R $\alpha$ expression in alveolar macrophages. (a) I/4ra mRNA expression analysis of primary alveolar macrophages from C57BL/6 wt or $\mathrm{LMP7}^{-1}$ mice polarized for $24 \mathrm{~h}$ with LPS/IFN $\gamma$ (M1) or IL-4 (M2), relative to Actb expression. Results are combined data from three independent experiments (mean + S.E.M., one-way ANOVA with Bonferroni's multiple comparison test; n.s., not significant). (b) IL4R $\alpha$ expression within 0-180 min after IL-4 treatment in primary alveolar macrophages from $\mathrm{C} 57 \mathrm{BL} / 6$ wt or $\mathrm{LMP7}^{-/-}$mice and densitometric analysis (the same membrane and thus $\beta$-Actin loading control was used for Figure 4d)

knockout animals..$^{9,12,37,38}$ In order to exclude that the presence of mixed immunoproteasomes in LMP7-deficient alveolar macrophages accounts for augmented IL-4 signaling and M2 polarization, we specifically inhibited the catalytic activity of LMP7 in alveolar macrophages using the LMP7specific inhibitor ONX-0914 and assayed for M2 polarization. This approach also allowed us to investigate the druggability of immunoproteasome-dependent alternative activation of alveolar macrophages. ONX-0914 is the first of a novel class of site-specific immunoproteasome inhibitors that preferentially binds to the LMP7 catalytic site as demonstrated by crystal structure analysis of mouse immunoproteasomes ${ }^{4}$ and active site-based ELISA. ${ }^{6}$ It has been shown to provide potent in vivo anti-autoimmune activity in several mouse models. ${ }^{6,5}$ Here, we used an activity-based pulldown to prove increased binding specificity of ONX-0914 to LMP7 over $\beta 5$ and thus preferential inhibition of LMP7. Importantly, pretreatment of primary as well as MH-S cells with ONX-0914 increased IL-4 triggered M2 polarization. Again, IL4Ra expression and STAT6 signaling were considerably augmented by impairment of LMP7 function. These results not only replicate our findings with alveolar macrophages of LMP7-deficient mice and reveal
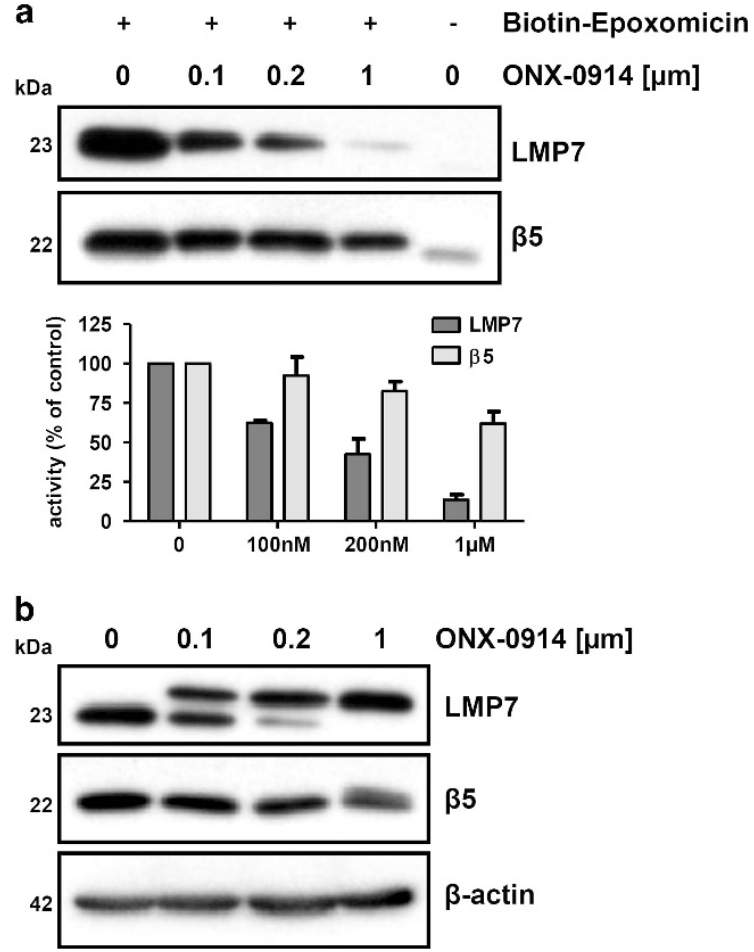

Figure 7 Activity-based inhibitor profiling of immunoproteasome LMP7-specific inhibitor ONX-0914. (a) Lysates of ONX-0914 inhibitor-treated MH-S cells (1 h) or DMSO-treated controls were incubated with pan-reactive proteasome ABP biotinepoxomycin or DMSO (vehicle control). Biotin ABP-labeled active subunits were affinity purified and visualized by western blot immunodetection of catalytic immunoproteasome subunit LMP7 and standard proteasome subunit $\beta 5$. Western blots are representative for three independent experiments, densitometric analysis shows combined results (mean+S.E.M.) of three independent experiments (activity of control is set to $100 \%$ ). (b) Western blot of ONX-0914-treated lysates before ABP incubation reveals a mobility shift in LMP7 protein when ONX-0914 is bound. $\beta$-Actin served as loading control, results are representative for three independent experiments

a novel role for the immunoproteasome in innate immune cell function but also provide proof-of-concept evidence for the specific therapeutic targeting of LMP7-specific proteasome activity for regulating alternative activation of alveolar macrophages.

Polarization of alveolar macrophages in lung disease. Since the initial recognition of IL-4-mediated alternative macrophage activation, ${ }^{39,40}$ numerous studies have investigated polarization mainly of bone marrow- and monocytederived macrophages. The finding that tissue macrophages are of different origin and self-renew throughout life, ${ }^{41-43}$ however, has stimulated research on the polarization capability of alveolar macrophages as a specific set of tissue-resident macrophages. ${ }^{23}$

The essential role of alveolar macrophage plasticity for the maintenance of respiratory health is well established. ${ }^{23,27}$ Proinflammatory M1 alveolar macrophages not only fight invading pathogens but also contribute to tissue damage of infected airways. ${ }^{44}$ In a similar manner, M2-polarized cells are not only required for proper resolution of airway inflammation or pulmonary wound healing, ${ }^{16}$ but excessive $\mathrm{M} 2$ activity also 
a

$\frac{5 \mathrm{~h} \text { ONX-0914 }}{180 \mathrm{~min} \frac{+\mathrm{IL}-4}{60 \mathrm{~min} \square}}$

$30 \min \square$

$15 \min \square$ c
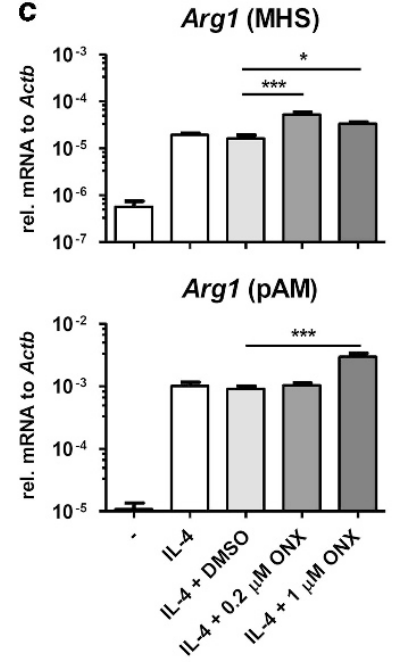

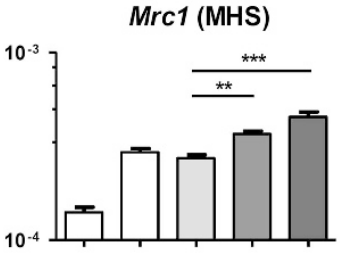

Mrc1 (pAM)

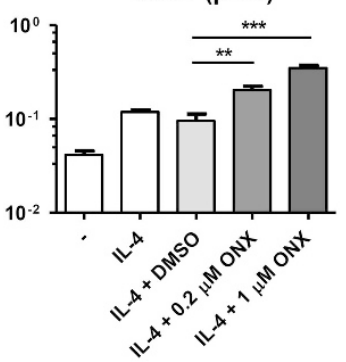

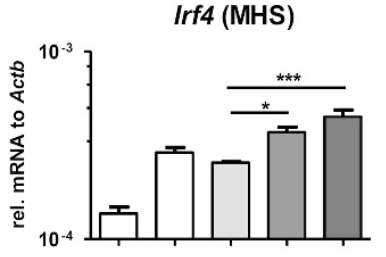

Irf4 (pAM)

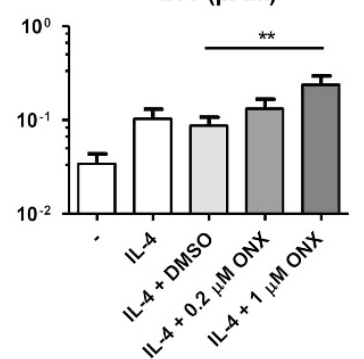

b
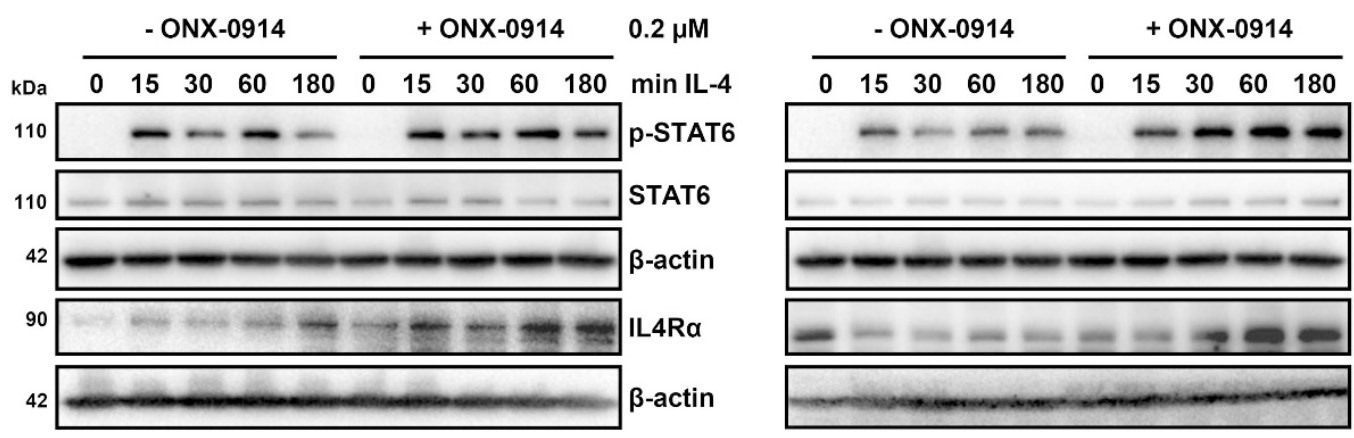

$1 \mu \mathrm{M}$

$\min$ IL-4

p-STAT6

STAT6

$\beta$-actin

IL4Ra

$\beta$-actin

Figure 8 LMP7 inhibition augments M2 polarization in alveolar macrophages. (a) Treatment scheme for (b): MH-S cells were incubated for $5 \mathrm{~h}$ with ONX-0914 or DMSO, IL-4 was added after different time points for the indicated durations. All samples were analyzed after $5 \mathrm{~h}$. (b) Time course of STAT6 (pTyr ${ }^{641}$ ) pathway activation and IL4R $\alpha$ expression within 0-180 min after IL-4 treatment in MH-S with or without ONX-0914 pretreatment (0.2 or $1 \mu \mathrm{M})$. Results are representative for three independent experiments. (c) M2 marker gene expression (Arg1, Mrc1 and Irf4) in MH-S cells or primary alveolar macrophages (pAM) from C57BL/6 mice: cells were pretreated with DMSO or ONX-0914 (0.2 or $1 \mu \mathrm{M})$ for $2 \mathrm{~h}$, afterward IL-4 was added for another $4 \mathrm{~h}$. Untreated cells and cells treated only with IL-4 served as controls. Shown are the mean+S.E.M. of three individual experiments (MH-S) or 3-6 individual mice (pAM). One-way ANOVA with Bonferroni's multiple comparison test $\left({ }^{\star} P<0.05,{ }^{* \star} P<0.01,{ }^{* \star *} P<0.001\right)$

contributes to the pathology of chronic lung diseases characterized by increased tissue remodeling as evidenced for asthma but also suggested recently for pulmonary fibrosis. ${ }^{45-47}$ Specifically, isolated macrophages obtained from bronchoalveolar lavage (BAL) of patients with idiopathic pulmonary fibrosis reveal an alternatively activated phenotype, produce high levels of the Th2-related chemokine CCL18 and stimulate fibroblast collagen production. ${ }^{25}$ In contrast, in the Th2-driven inflammation and fibrosis model of helminth infection, alternatively activated macrophages have been shown to counterbalance acute inflammation, inhibit Th2 cell differentiation and cytokine production thereby contributing to reduced fibrosis. ${ }^{48,49}$ The specific role of alternatively activated macrophages thus appears to be disease-dependent and may be further influenced by the origin of the macrophages as tissue-resident alveolar macrophages or inflammatory blood-derived macrophages. In support of this notion, inflammatory monocytes facilitate progression of pulmonary fibrosis, whereas alternatively activated lung macrophages have a resolution-promoting potency during the reversible phase of bleomycin-induced pulmonary fibrosis. ${ }^{24}$ In the same model, the inhibitory cell surface receptor, paired Ig-like receptor B (PIR-B), which is abundantly expressed on myeloid cells of fibrotic lungs, was shown to inhibit pulmonary fibrosis and alternative macrophage polarization, suggesting that PIR-B inhibits the profibrogenic properties of alternatively activated alveolar macrophages. ${ }^{50}$ In this context, recent research indicates that M2-skewed alternative activity of lung macrophages and in particular, M2-mediated induction of CCL17, enhances their profibrotic program, and/or causes a Th2-prone pathological setting thus paving the way for pulmonary fibrosis or asthma. ${ }^{51-53}$ Augmentation of alternative macrophage activation by immunoproteasome dysfunction most likely has important consequences for homeostasis and response to environmental stimuli of the pulmonary tissue. Intriguingly, there is not much known about regulation of immunoproteasome expression and activity in respiratory disease. A recent study suggested elevated expression of immunoproteasome subunits in alveolar macrophages in patients with sarcoidosis. ${ }^{54}$ Preliminary data of our group also suggest increased protein levels of LMP7 in BAL cells isolated from fibrotic lungs of bleomycin instilled mice. Future studies using LMP7-deficient mice will investigate the functional relevance 
of LMP7 activity for the course of bleomycin-triggered pulmonary fibrosis. Immunoproteasome function in macrophages has also been reported to be affected by alcohol and virus infection. ${ }^{55}$ Our own data indicate downregulation of immunoproteasome function in the lung by cigarette smoke. ${ }^{56}$ These data support the notion that immunoproteasome function in macrophages is affected by environmental stimuli and possibly altered in the course of pulmonary fibrosis. Promoting pro-resolving M2 macrophage polarization represents a promising strategy to alleviate excessive inflammatory conditions as exemplified by corticosteroid treatment of patients with mild chronic obstructive pulmonary disease in case of acute exacerbations. ${ }^{57-59}$ Under more severe inflammatory conditions of acute respiratory distress syndrome (ARDS) and acute lung injury, however, therapeutic application of steroids remains controversial as these drugs have no benefits and are associated with adverse outcome during late stages of ARDS. ${ }^{59,60}$ Alternative strategies of macrophage modulation that protect against acute lung injury include deletion of Akt2 kinase to effectively enhance M2 polarization of alveolar macrophages in mice while completely abrogating M1 polarization. ${ }^{61}$ However, protection was limited to aseptic ARDS conditions, as the complete blocking of classical macrophage activation impaired innate immune response of alveolar macrophages to live bacteria, which is required for effective bacterial clearance. Therapeutic strategies that augment M2 activation but retain M1 plasticity may thus be more favorable for the treatment of acute lung injury. Immunoproteasome-specific inhibition may represent according to our results a more favorable approach for the treatment of pneumonia- or sepsis-related ARDS.

\section{Materials and Methods}

Animals. BALB/CAnCrl wt or C57BL/6J wt were from Charles River Laboratories, Kißlegg, Germany. LMP7 ${ }^{-1-}\left(\mathrm{Psmb}^{\mathrm{tm} 1 \mathrm{Hif}}\right)^{62}$ mice with C57BL/6J background were bred at the Helmholtz Zentrum München. Eight- to 12-week-old mice were sacrificed for BAL preparation. All animal procedures were conducted with approval of the local ethics committee and the Bavarian Animal Research Authority of Germany

Alveolar macrophage cell line. Murine alveolar macrophage cell line (MH-S, derived from BALB/C) was purchased from American Type Culture Collection (Manassas, VA, USA, ATCC no. CRL-2019). Cells were grown in complete RPMI-1640 medium supplemented with $10 \%$ fetal bovine serum (Biochrom, Berlin, Germany) and $50 \mu \mathrm{M} \beta$-mercaptoethanol and $100 \mathrm{U} / \mathrm{ml}$ penicillin and streptomycin (Life Technologies, Paisley, Scotland, UK) at $37^{\circ} \mathrm{C}$ and $5 \% \mathrm{CO}_{2}$. MH-S cells were plated on 6-, 12- or 24-well plates with a density of $3 \times 10^{5}-6 \times 10^{5}$ cells $/ \mathrm{cm}^{2}$ the day before treatment.

Isolation of primary resident alveolar macrophages. Primary alveolar macrophages were isolated from the lungs of mice by BAL with eight washes of $1 \mathrm{ml}$ PBS at room temperature. Cells were pelleted for $5 \mathrm{~min}$ at 1500 r.p. $\mathrm{m}$. and washed twice in complete RPMI- 1640 medium. In all, $1 \times 10^{5}-5 \times 10^{5}$ cells were seeded in 12- or 24-well plates and were allowed to adhere for $1-3 \mathrm{~h}$. Non-adherent cells were removed by washing two times with PBS.

Polarization of alveolar macrophages into M1 or M2 phenotype. Primary alveolar macrophages and MH-S cells were polarized toward the M1 phenotype with LPS $(1 \mu \mathrm{g} / \mathrm{ml}$, E. coli O55:B5, strain CDC 1644-70, Sigma-Aldrich, St. Louis, MO, USA) and/or IFN $\gamma$ (20 ng/ml, Immunotool, Friesoythe, Germany) or toward the M2 phenotype with IL-4 or IL-13 (20 ng/ml, Immunotool) treatment for up to $72 \mathrm{~h}$. Unpolarized cells (M0) served as controls. Cell culture supernatants were collected for measurement of CCL17. Adherent alveolar macrophages were washed twice with PBS and harvested for total RNA isolation or protein extraction.
Treatment with proteasome inhibitor ONX-0914. For proteasome inhibitor studies, MH-S cells or primary alveolar macrophages were pretreated with ONX-0914 (APExBIO, dissolved in DMSO, Houston, TX, USA) for $2 \mathrm{~h}$ to $4 \mathrm{~h} 45 \mathrm{~min}$, time-matched controls were treated with DMSO only. Afterward, IL-4 was added to the cell culture medium while ONX-0914 was still present.

WST-cell viability assay. Viability testing of $24 \mathrm{~h}$ polarized primary wt and $\mathrm{LMP}^{-1-}$ alveolar macrophages or ONX-0914-treated MH-S cells (6 or $24 \mathrm{~h}$ ) was performed in a 96-well format with 30000 cells per well with the WST-1 assay (Roche, Unterhaching, Germany) according to the manufacturer's instructions.

RNA extraction and quantitative RT-PCR analysis. Total RNA was extracted from alveolar macrophages using Quick-RNA MicroPrep kit (ZYMO, Freiburg, Germany). 50-1000 ng total RNA was used for cDNA synthesis by Superscript II Reverse Transcriptase kit with the protocol described previously. ${ }^{63}$ To determine the expression of target genes relative to the actin housekeeper Actb, the ABsolute QPCR SYBR Green ROX Mix (Thermo Scientific, Wilmington, DE, USA) was used on an ABI PRISM 7000 detection system (Applied Biosystems, Foster City, CA, USA). Primer sequences are given in Supplementary Table I. Relative expression of target genes and housekeeping gene Actb was calculated according to the $2^{-\Delta C t}$ method. $^{63}$

\section{Transcriptome analysis}

RNA isolation: Total RNA was isolated using the RNeasy Mini (Qiagen, Hilden, Germany) including RNase-Free DNase for digestion of remaining genomic DNA. The Agilent 2100 Bio-analyzer (Santa Clara, CA, USA) was used to assess RNA quality and only high-quality RNA (RIN $>7$ ) was used for microarray analysis. Expression profiling: Total RNA (about $10 \mathrm{ng}$ ) was amplified using the Ovation PicoSL WTA System V2 in combination with the Encore Biotin IL Module (Nugen, San Carlos, CA, USA). In all, $1000 \mathrm{ng}$ of amplified CDNA was hybridized to Mouse Ref-8 v2.0 Expression Bead Chips (Illumina, San Diego, CA, USA). Staining and scanning were done according to the Illumina expression protocol. Data were processed using the GenomeStudioV2011.1 software (San Diego, CA, USA; gene expression module version 1.9.0) in combination with the Mouse Ref8-V2_0_R3_11278551_A.bgx annotation file. The background subtraction option was used and an offset to remove remaining negative expression values was introduced. CARMAweb was used for quantile normalization. ${ }^{64}$

Statistical transcriptome analysis: Statistical analyses were performed by utilizing the statistical programming environment $R$ (R Development Core Team, free software: https://www.r-project.org/). Gene-wise testing for differential expression was done using the limma $t$-test $(P<0.05)$. Volcano plot, heat map and GO enrichment analysis were done with $\mathrm{R} /$ Biocondcutor (www.bioconductor.org).

GSEA analysis: For global transcriptome comparison, GSEA was performed as follows. ${ }^{65}$ All genes assessed by microarray were ranked and weighted by their mean log2 fold change. Gene set termed 'PID_IL4_2PATHWAY' was downloaded from GSEA website. These data were then analyzed using the GSEA v2.0.13 tool (free software: http://software.broadinstitute.org/gsea/index.jsp). Array data have been submitted to GEO (GSE72000).

Gene set enrichment was tested between IL-4 regulated genes of LMP7 $7^{-1-}$ and wt samples, using the gene expression signature of IL-4 signaling defined by the Broad Institute (http://www.broadinstitute.org/). The heat map shows the mean intensity of the transcripts of the gene set 'LU_IL4 signaling' for each sample, clustered by the hierarchical method to reflect the degree of similarity.

Cell lysis and western blot. For protein isolation, alveolar macrophages were washed twice with ice cold PBS and lysed with RIPA buffer ( $50 \mathrm{mM}$ Tris. $\mathrm{HCl}$, pH 7.5, $150 \mathrm{mM} \mathrm{NaCl}, 1 \%$ Nonidet P-40, $0.5 \%$ sodium deoxycholate and $0.1 \%$ SDS). Samples were centrifuged to remove cell debris and protein concentrations were determined using standard Bradford assays. In all, $10 \mu \mathrm{g}$ of protein lysates were separated on $10 \%$ SDS-PAGE and blotted onto polyvinylidene difluoride membranes (162-0177, Bio-Rad, Hercules, CA, USA). The following antibodies were used: LMP2 (ab3328, Abcam, Cambridge, UK), LMP7 (ab3329, Abcam), 20S $\alpha 1+2+3+5+6+7$ (hereafter called $\alpha 1-7$; clone MCP231, ab2267, Abcam), Psmb5 (detecting $\beta 5$, ab90867, Abcam), AKT (4685, Cell Signaling, Beverly, MA, USA), p-AKT (4060, pSer $^{473}$, Cell Signaling), STAT6 (9362, Cell Signaling), p-STAT6 (9361, pTyr ${ }^{641}$, Cell Signaling), IRF4 (M17, Santa Cruz, Dallas, TX, USA), Arginase 1 (H-52, Santa Cruz), iNOS (610331, BD Transduction Laboratories, Franklin Lakes, NJ, USA), IL4R (ab157162, Abcam), HRP-conjugated anti- $\beta$-actin (Sigma-Aldrich), HRP-conjugated anti-rabbit (Abcam) and anti-goat antibodies (Santa Cruz). 
ABP labeling of proteasomes. Activity of the constitutive and immunoproteasome subunits was monitored by using a set of $\mathrm{ABPs}^{66}$ as previously described. ${ }^{13}$

Activity-based pulldown. In order to determine the specificity of the LMP7 inhibitor ONX-0914, we performed ABP-based pulldowns. For that, MH-S cells were treated with indicated concentrations of LMP7 inhibitor ONX-0914 or DMSO only (vehicle control) for $1 \mathrm{~h}$. Cells were then harvested by trypsination, and the cell pellets were resuspended in TSDG buffer $(10 \mathrm{mM}$ Tris/ $/ \mathrm{HCl}, 1.1 \mathrm{mM} \mathrm{MgCl}, 10 \mathrm{mM}$ EDTA, $1 \mathrm{mM} \mathrm{NaN}$, $1 \mathrm{mM}$ DTT, $2 \mathrm{mM}$ ATP, 10\% (v/v) glycerol, pH 7.0) containing complete protease inhibitor (Roche) and directly lysed by repeated $(7 x)$ freeze and thawing steps in liquid nitrogen or in a water bath at RT, respectively. After centrifugation for $20 \mathrm{~min}$ at $14000 \times \mathrm{g}$, protein concentration was determined using BCA Protein Assay (Bio-Rad) and samples were stored at $-80^{\circ} \mathrm{C}$. Cell lysates were defrosted and $0.5 \mathrm{mg}$ of total protein was adjusted to a final concentration of $1 \mathrm{mg} / \mathrm{ml}$ with $50 \mathrm{mM}$ HEPES (pH 7.4). In all, $5 \mu \mathrm{M}$ biotin-epoxomicin ABP (from $100 \times$ DMSO stock; provided by Hermen Overkleeft ${ }^{32}$ ) or DMSO was added and incubated for $2 \mathrm{~h}$ at $37^{\circ} \mathrm{C}$ with 600 r.p.m. shaking. Depletion of unbound $A B P$ was done by size exclusion using PD MidiTrap G-25 columns (GE Healthcare, Freiburg, Germany) according to the manufacturer's recommendations. Eluted samples were denatured by $1 \%$ SDS at $95^{\circ} \mathrm{C}$ for 8 min. Samples were centrifuged for 1 min $10000 \times g$ at RT and diluted 1:50 in $50 \mathrm{mM}$ HEPES (pH 7.4). Each $40 \mu \mathrm{l}$ of StrepTactin Superflow $50 \%$ bead slurry (IBA, Goettingen, Germany), prewashed with $50 \mathrm{~m}$ HEPES ( $\mathrm{pH} 7.4$ ), was added to the sample and incubated for $2 \mathrm{~h}$ at $4{ }^{\circ} \mathrm{C}$ on a rotator, then was washed three times with PBS. Bound proteins were eluted by boiling the beads for $15 \mathrm{~min}$ at $95^{\circ} \mathrm{C}$ in $60 \mu \mathrm{l} 1 \times$ Laemmli buffer and stored at $-20{ }^{\circ} \mathrm{C}$ for further analysis. For immunodetection of proteasome subunits LMP7 and $\beta 5,25 \mu \mathrm{l}$ of the elution fractions were separated on a $15 \%$ polyacrylamide gel for immunoblotting.

Chemokine analysis by ELISA. Cell culture supernatant of alveolar macrophages was collected after treatment with LPS/IFN $\gamma$ and IL-4 for M1 and M2 polarization and stored at $-80^{\circ} \mathrm{C}$ until analysis. CCL17 concentration was measured using a specific ELISA (Duoset Detection Kit; R\&D Systems, Minneapolis, MN, USA) according to the manufacturer's instructions. Concentrations were calculated with a standard curve (detection limit $31 \mathrm{pg} / \mathrm{ml}$ ).

Statistics and software. Data were analyzed with ImageLab (Bio-Rad), ImageJ (imagej.nih.gov/ij) or Prism5 (GraphPad Software, Inc., La Jolla, CA, USA). Statistics were performed using Prism5 with parametric tests and appropriate post hoc-analysis, details for each analysis are given in the figure legends. $P$-values $<0.05$ were considered statistically significant.

\section{Conflict of Interest}

The authors declare no conflict of interest.

Acknowledgements. We thank David Kutschke and Anna Fuchs for excellent technical assistance. Financial support was provided by China Scholarship Counci (CSC). SC is the recipient of a fellowship of CSC (2010624122). IEK was funded by the Helmholtz Graduate School 'Lung Biology and Disease' at the Comprehensive Pneumology Center.

1. Groll M, Ditzel L, Löwe J, Stock D, Bochtler M, Bartunik HD et al. Structure of $20 \mathrm{~S}$ proteasome from yeast at 2.4A resolution. Nature 1997; 386: 463-471.

2. Groettrup M, Kirk CJ, Basler M. Proteasomes in immune cells: more than peptide producers? Nat Rev Immunol 2010; 10: 73-78.

3. Kloetzel PM, Ossendorp F. Proteasome and peptidase function in MHC-class-I-mediated antigen presentation. Curr Opin Immunol 2004; 16: 76-81.

4. Huber EM, Basler M, Schwab R, Heinemeyer W, Kirk CJ, Groettrup M et al. Immuno- and constitutive proteasome crystal structures reveal differences in substrate and inhibitor specificity. Cell 2012; 148: 727-738.

5. Basler M, Mundt S, Muchamuel T, Moll C, Jiang J, Groettrup M et al. Inhibition of the immunoproteasome ameliorates experimental autoimmune encephalomyelitis. EMBO Mol Med 2014; 6: 226-238.

6. Muchamuel T, Basler M, Aujay MA, Suzuki E, Kalim KW, Lauer C et al. A selective inhibitor of the immunoproteasome subunit LMP7 blocks cytokine production and attenuates progression of experimental arthritis. Nat Med 2009; 15: 781-787.
7. Hensley SE, Zanker D, Dolan BP, David A, Hickman HD, Embry AC et al. Unexpected role for the immunoproteasome subunit LMP2 in antiviral humoral and innate immune responses. $\mathrm{J}$ Immunol 2010; 184: 4115-4122.

8. Kalim KW, Basler M, Kirk CJ, Groettrup M. Immunoproteasome subunit LMP7 deficiency and inhibition suppresses Th1 and Th17 but enhances regulatory T cell differentiation. J Immunol 2012; 189: 4182-4193.

9. de Verteuil DA, Rouette A, Hardy M-P, Lavallée S, Trofimov A, Gaucher É et al. Immunoproteasomes shape the transcriptome and regulate the function of dendritic cells. $J$ Immunol 2014; 193: 1121-1132.

10. Reis J, Guan XQ, Kisselev AF, Papasian CJ, Qureshi AA, Morrison DC et al. LPS-induced formation of immunoproteasomes: TNF- $\alpha$ and nitric oxide production are regulated by altered composition of proteasome-active sites. Cell Biochem Biophys 2011; 60: 77-88.

11. Ebstein F, Kloetzel P-M, Krüger E, Seifert U. Emerging roles of immunoproteasomes beyond MHC class I antigen processing. Cell Mol Life Sci 2012; 69: 2543-2558.

12. Basler M, Kirk CJ, Groettrup M. The immunoproteasome in antigen processing and other immunological functions. Curr Opin Immunol 2013; 25: 74-80.

13. Keller IE, Vosyka O, Takenaka S, Kloß A, Dahlmann B, Willems LI et al. Regulation of immunoproteasome function in the lung. Sci Rep 2015; 5: 10230.

14. Lambrecht BN. Alveolar macrophage in the driver's seat. Immunity 2006; 24: 366-368.

15. Guth AM, Janssen WJ, Bosio CM, Crouch EC, Henson PM, Dow SW. Lung environment determines unique phenotype of alveolar macrophages. Am J Physiol Lung Cell Mol Physiol 2009; 296: L936-L946.

16. Alber A, Howie SEM, Wallace WAH, Hirani N. The role of macrophages in healing the wounded lung. Int J Exp Pathol 2012; 93: 243-251.

17. Aggarwal NR, King LS, D'Alessio FR. Diverse macrophage populations mediate acute lung inflammation and resolution. Am J Physiol Lung Cell Mol Physiol 2014; 306: L709-L725.

18. Mills CD, Kincaid K, Alt JM, Heilman MJ, Hill AM. M-1/M-2 macrophages and the Th1/Th2 paradigm. J Immunol 2000; 164: 6166-6173.

19. Redente EF, Higgins DM, Dwyer-Nield LD, Orme IM, Gonzalez-Juarrero M, Malkinson AM. Differential polarization of alveolar macrophages and bone marrow-derived monocytes following chemically and pathogen-induced chronic lung inflammation. J Leukoc Biol 2010; 88: $159-168$.

20. Krysko DV, D'Herde K, Vandenabeele P. Clearance of apoptotic and necrotic cells and its immunological consequences. Apoptosis 2006; 11: 1709-1726.

21. Gordon S. Alternative activation of macrophages. Nat Rev Immunol 2003; 3: 23-35.

22. Lawrence T, Natoli G. Transcriptional regulation of macrophage polarization: enabling diversity with identity. Nat Rev Immunol 2011; 11: 750-761.

23. Hussell T, Bell TJ. Alveolar macrophages: plasticity in a tissue-specific context. Nat Rev Immunol 2014; 14: 81-93.

24. Gibbons MA, MacKinnon AC, Ramachandran P, Dhaliwal K, Duffin R, Phythian-Adams AT et al. Ly6Chi monocytes direct alternatively activated profibrotic macrophage regulation of lung fibrosis. Am J Respir Crit Care Med 2011; 184: 569-581.

25. Prasse A, Pechkovsky DV, Toews GB, Jungraithmayr W, Kollert F, Goldmann T et al. A vicious circle of alveolar macrophages and fibroblasts perpetuates pulmonary fibrosis via CCL18. Am J Respir Crit Care Med 2006; 173: 781-792.

26. Huaux F, Liu T, McGarry B, Ullenbruch M, Phan SH. Dual roles of IL-4 in lung injury and fibrosis. J Immunol 2003; 170: 2083-2092.

27. Sica A, Mantovani A. Macrophage plasticity and polarization: in vivo veritas. J Clin Invest 2012; 122: 787-795.

28. Satoh T, Takeuchi O, Vandenbon A, Yasuda K, Tanaka Y, Kumagai Y et al. The Jmjd3-Irf4 axis regulates M2 macrophage polarization and host responses against helminth infection. Nat Immunol 2010; 11: 936-944.

29. Nakamura A, Ebina-Shibuya R, Itoh-Nakadai A, Muto A, Shima $H$, Saigusa $D$ et al. Transcription repressor Bach2 is required for pulmonary surfactant homeostasis and alveolar macrophage function. J Exp Med 2013; 210: 2191-2204.

30. Nelms K, Keegan AD, Zamorano J, Ryan JJ, Paul WE. The IL-4 receptor: signaling mechanisms and biologic functions. Annu Rev Immunol 1999; 17: 701-738.

31. Huber EM, Groll M. Inhibitors for the immuno- and constitutive proteasome: current and future trends in drug development. Angew Chem Int Ed Engl 2012; 51: 8708-8720.

32. Li N, Kuo C-L, Paniagua G, van den Elst H, Verdoes M, Willems LI et al. Relative quantification of proteasome activity by activity-based protein profiling and LC-MS/MS. Nat Protoc 2013; 8: 1155-1168.

33. Tosello V, Zamarchi R, Merlo A, Gorza M, Piovan E, Mandruzzato S et al. Differential expression of constitutive and inducible proteasome subunits in human monocyte-derived DC differentiated in the presence of IFN-alpha or IL-4. Eur J Immunol 2009; 39: 56-66.

34. Semren N, Welk V, Korfei M, Keller IE, Fernandez IE, Adler $\mathrm{H}$ et al. Regulation of $26 \mathrm{~S}$ proteasome activity in pulmonary fibrosis. Am J Respir Crit Care Med 2015; 192 : 1089-1101.

35. Jang ER, Lee N-R, Han S, Wu Y, Sharma LK, Carmony KC et al. Revisiting the role of the immunoproteasome in the activation of the canonical NF- $\kappa$ B pathway. Mol Biosyst 2012; 8: 2295-2302.

36. Wei Q, Sha Y, Bhattacharya A, Abdel Fattah E, Bonilla D, Jyothula SSSK et al. Regulation of IL-4 receptor signaling by STUB1 in lung inflammation. Am J Respir Crit Care Med 2014; 189: $16-29$. 
37. Griffin TA. Immunoproteasome assembly: cooperative incorporation of interferon gamma (IFN-gamma)-inducible subunits. J Exp Med 1998; 187: 97-104.

38. Dahlmann B, Ruppert T, Kuehn L, Merforth S, Kloetzel PM. Different proteasome subtypes in a single tissue exhibit different enzymatic properties. J Mol Biol 2000; 303 643-653.

39. Stein M, Keshav S, Harris N, Gordon S. Interleukin 4 potently enhances murine macrophage mannose receptor activity: a marker of alternative immunologic macrophage activation. J Exp Med 1992; 176: 287-292.

40. Abramson SL, Gallin Jl. IL-4 inhibits superoxide production by human mononuclear phagocytes. J Immunol 1990; 144: 625-630.

41. Guilliams M, De Kleer I, Henri S, Post S, Vanhoutte L, De Prijck S et al. Alveolar macrophages develop from fetal monocytes that differentiate into long-lived cells in the first week of life via GM-CSF. J Exp Med 2013; 210: 1977-1992.

42. Hashimoto D, Chow A, Noizat C, Teo P, Beasley MB, Leboeuf M et al. Tissue-resident macrophages self-maintain locally throughout adult life with minimal contribution from circulating monocytes. Immunity 2013; 38: 792-804.

43. Yona S, Kim K-W, Wolf Y, Mildner A, Varol D, Breker M et al. Fate mapping reveals origins and dynamics of monocytes and tissue macrophages under homeostasis. Immunity 2013; 38: 79-91.

44. Högner K, Wolff T, Pleschka S, Plog S, Gruber AD, Kalinke U et al. Macrophage-expressed IFN- $\beta$ contributes to apoptotic alveolar epithelial cell injury in severe influenza virus pneumonia. PLoS Pathog 2013; 9: e1003188.

45. Mantovani A, Biswas SK, Galdiero MR, Sica A, Locati M. Macrophage plasticity and polarization in tissue repair and remodelling. J Pathol 2013; 229: 176-185.

46. Wynn TA, Barron L. Macrophages: master regulators of inflammation and fibrosis. Semin Liver Dis 2010; 30: 245-257.

47. Pulichino A-M, Wang I-M, Caron A, Mortimer J, Auger A, Boie $Y$ et al. Identification of transforming growth factor beta1-driven genetic programs of acute lung fibrosis. Am J Respir Cell Mol Biol 2008; 39: 324-336.

48. Herbert DR, Hölscher C, Mohrs M, Arendse B, Schwegmann A, Radwanska M et al. Alternative macrophage activation is essential for survival during schistosomiasis and downmodulates T helper 1 responses and immunopathology. Immunity 2004; 20: 623-635

49. Nair MG, Du Y, Perrigoue JG, Zaph C, Taylor JJ, Goldschmidt M et al. Alternatively activated macrophage-derived RELM-\{alpha\} is a negative regulator of type 2 inflammation in the lung $J$ Exp Med 2009; 206: 937-952.

50. Karo-Atar D, Moshkovits I, Eickelberg O, Königshoff M, Munitz A. Paired immunoglobulin-like receptor-B inhibits pulmonary fibrosis by suppressing profibrogenic properties of alveolar macrophages. Am J Respir Cell Mol Biol 2013; 48: 456-464.

51. Pechkovsky DV, Prasse A, Kollert F, Engel KMY, Dentler J, Luttmann W et al. Alternatively activated alveolar macrophages in pulmonary fibrosis-mediator production and intracellular signal transduction. Clin Immunol 2010; 137: 89-101.
52. Belperio JA, Dy M, Murray L, Burdick MD, Xue YY, Strieter RM et al. The role of the Th2 CC chemokine ligand CCL17 in pulmonary fibrosis. J Immunol 2004; 173: 4692-4698.

53. Staples KJ, Hinks TSC, Ward JA, Gunn V, Smith C, Djukanović R. Phenotypic characterization of lung macrophages in asthmatic patients: overexpression of CCL17. J Allergy Clin Immunol 2012; 130: 1404-1412.e7.

54. Sixt SU, Costabel U, Bonella F, Grunert K, Alami R, Hakenbeck J et al. Alveolar and intraparenchymal proteasome in sarcoidosis. Respir Med 2014; 108: 1534-1541.

55. Haorah J, Heilman D, Diekmann C, Osna N, Donohue TM, Ghorpade A et al. Alcohol and HIV decrease proteasome and immunoproteasome function in macrophages: implications for impaired immune function during disease. Cell Immunol 2004; 229: 139-148.

56. Kammerl IE, Dann A, Mossina A, Dorothee B, Lukas C, Vosyka O et al. Impairment of immunoproteasome function by cigarette smoke and in COPD. Am J Respir Crit Care Med; doi:10.1164/rccm.201506-11220C; advance online publication, 12 January 2016; http:// www.atsjournals.org/doi/abs/10.1164/rccm.201506-1122OC\#.VqvFdLLhBpg.

57. Hao N-B, Lü M-H, Fan Y-H, Cao Y-L, Zhang Z-R, Yang S-M. Macrophages in tumor microenvironments and the progression of tumors. Clin Dev Immunol 2012; 2012: 948098

58. Woods JA, Wheeler JS, Finch CK, Pinner NA. Corticosteroids in the treatment of acute exacerbations of chronic obstructive pulmonary disease. Int $\mathrm{J}$ Chron Obstruct Pulmon Dis 2014; 9: 421-430.

59. Hough CL. Steroids for acute respiratory distress syndrome? Clin Chest Med 2014; 35 781-795.

60. Ruan S-Y, Lin H-H, Huang C-T, Kuo P-H, Wu H-D, Yu C-J. Exploring the heterogeneity of effects of corticosteroids on acute respiratory distress syndrome: a systematic review and meta-analysis. Crit Care 2014; 18: R63.

61. Vergadi E, Vaporidi K, Theodorakis EE, Doxaki C, Lagoudaki E, leronymaki E et al. Akt2 deficiency protects from acute lung injury via alternative macrophage activation and miR-146a induction in mice. J Immunol 2014; 192: 394-406.

62. Fehling H, Swat W, Laplace C, Kuhn R, Rajewsky K, Muller U et al. MHC class I expression in mice lacking the proteasome subunit LMP-7. Science 1994; 265: 1234-1237.

63. Yin R, Tian F, Frankenberger B, de Angelis MH, Stoeger T. Selection and evaluation of stable housekeeping genes for gene expression normalization in carbon nanoparticle-induced acute pulmonary inflammation in mice. Biochem Biophys Res Commun 2010; 399: 531-536.

64. Rainer J, Sanchez-Cabo F, Stocker G, Sturn A, Trajanoski Z. CARMAweb: comprehensive R- and bioconductor-based web service for microarray data analysis. Nucleic Acids Res 2006; 34: W498-W503.

65. Subramanian A, Tamayo P, Mootha VK, Mukherjee S, Ebert BL, Gillette MA et al. Gene se enrichment analysis: a knowledge-based approach for interpreting genome-wide expression profiles. Proc Natl Acad Sci USA 2005; 102: 15545-15550.

66. Cravatt BF, Wright AT, Kozarich JW. Activity-based protein profiling: from enzyme chemistry to proteomic chemistry. Annu Rev Biochem 2008; 77: 383-414.

Supplementary Information accompanies this paper on Cell Death and Differentiation website (http://www.nature.com/cdd) 